\title{
EDITORIAL
}

\section{Forensic Analytical Chemistry: Connecting Science and Justice}

\author{
Márcia Andreia Mesquita Silva da Veiga (iD $\square$ \\ Guest Editor of this special edition on Forensic Analytical Chemistry \\ Associate Professor in the Department of Chemistry at the Faculty of Philosophy, Sciences and Letters \\ of Ribeirão Preto, University of São Paulo (FFCLRP-USP), Ribeirão Preto, SP, Brazil
}

Currently, analytical chemistry is more than simply its division into classical and instrumental. It is an interdisciplinary area that involves notions of biology, toxicology, statistics, computer science, and physics, among others. There are several areas of knowledge applied in the development of a chemical analysis, which is configured as all the processes necessary for the identification and quantification of the different components of a sample. When this sample is a trace material from a crime scene, analytical chemistry assumes a central role in the conversion of this sample into material evidence with legal value through consolidated and validated procedures, obtained by exhaustive investigative and methodological studies. The responsibility assumed is in the confidence of the result obtained, which will only be possible with the validation of the method. Although not all methods are perfect, a quantitative determination requires a precise and accurate methodology. Therefore, analytical chemistry is very important to forensic chemistry. Material evidence has a great influence on a trial because it is clothed in technical characteristics, and the expectation is that it will help to unequivocally clarify the truth of the facts. It is this expectation that makes the work of the analytical chemist so important in conducting an analytical procedure for forensic purposes. The result obtained may or may not incriminate someone.

Another analytical challenge in forensic analysis is the collection and preparation of a sample that has a criminal trace profile. Such procedures should preserve as much of the criminal evidence as possible. At a crime scene, several samples can be considered evidence: soils, fibers, glass, gunshot residues, explosives, among others. Locard's principle of exchange states that whenever two objects come into contact, an exchange of materials occurs between them and, thus, a connection is established between the suspect and the crime scene or between the suspect and the victim based on the transfer of fragments of the materials. Once again, analytical rigor will play a relevant role in the preservation and experimental conduct of the traces. A failure in the analytical procedure may make it impossible to use a trace as material evidence in a court of law, jeopardizing its use in the conviction of the judge or jury. It is up to the forensic analysts to provide a result with credibility and legal security, i.e., to rigorously follow the analytical protocols.

Forensic research is dynamic. One example is the demand for analytical methods that encompass the wide variety of newly emerging psychoactive substances (NPS), formerly known as "designer drugs", which must continually be detected and catalogued. In the Interview in this volume, Dr. Barry Logan tells us about this challenge in his career. I want to register my special thanks to Prof. Dr. Bruno Martinis from the Department of Chemistry at the Faculty of Philosophy, Sciences and Letters of Ribeirão Preto, University of São Paulo (DQ-FFCLRP-USP), who mediated and made possible the interview for BrJAC of one of the most prominent scientists in the area.

Cite: da Veiga, M. A. M. S. Forensic Analytical Chemistry: Connecting Science and Justice. Braz. J. Anal. Chem., 2022, 9 (34), pp 1-2. doi: http://dx.doi.org/10.30744/brjac.2179-3425.editorial.mamsveiga.N34 
In his Point of View, Federal Criminal Expert Marcus Andrade tells us about the complexity of forensic chemical analysis of works of art. The analysis of the surface of a painting requires, besides the historical study of the piece, the physical-chemical characterization of its components through an interesting combination of non-destructive analytical techniques such as microscopy, X-ray fluorescence, infrared spectroscopy, among others, which are powerful tools in the process of authenticating a work of art. Nevertheless, the art market, due to the high added value, has attracted organized crime in money laundering in cases of active and passive corruption.

Prof. Dr. Jesus Antônio Velho, Federal Criminal Expert and lecturer at the DQ-FFCLRP-USP, addresses the new trends in analytical chemistry for the examination and interpretation of traces of crimes: the determination of the origin of seized drugs (chemical profile), the investigation of document fraud, and the analysis of the evaluation of works of art. Professor Jesus' letter is the result of his long experience in the field and his exceptional insight into the forensic sciences. The author of several books in the field, his text is a gift to us all.

We hope readers will enjoy this special edition of BrJAC. Forensic professionals, students, professors, and researchers seek to contribute to criminal justice more broadly through tireless dedication. When we apply forensic analytical chemistry to a crime scene, more than an expert report, we give voice to the victims, who, through their traces, reveal to us the truth of the facts.

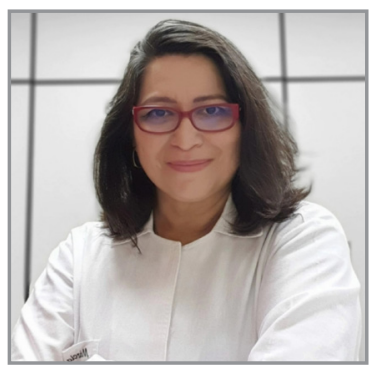

Márcia Andreia Mesquita Silva da Veiga is currently an Associate Professor in the Department of Chemistry at the Faculty of Philosophy, Sciences, and Letters of Ribeirão Preto, University of São Paulo, Brazil. She has a degree in Chemistry (Federal University of Amazonas, 1991), a master's in physical chemistry, and a doctoral in Analytical Chemistry (Federal University of Santa Catarina, 1996 and 2000), and post-Doc in Analytical Chemistry at the Institute of Chemistry, University of São Paulo (2005). Leads the research group L.Q.A.I.A. (Laboratory of Applied Instrumentation and Analytical Chemistry), and nowadays, is coordinator of the professional master's degree in Chemistry (PROFQUI) at the University of São Paulo. She works mainly with optical techniques for trace and isotopic analysis. Her current research focus is on sample preparation procedures, detection and quantification of nanomaterials and their application, bioaccessibility assays in foods and soils, the potential of high-resolution graphite furnace molecular absorption spectrometry for elemental and isotopic analysis, detection and quantification of gunshot residues, and new technological approaches to chemistry teaching. 